\title{
High Accuracy Low Cost RTKISINS for Land-Vehicles
}

\author{
Yayang Cheng ${ }^{1, a}$, Tongyue Gao ${ }^{2, b}$ and Shihao Zhu $^{2, c}$ \\ ${ }^{1}$ School of Mechatronic Engineering and Automation, Shanghai University, Shanghai 200072, \\ China; \\ ${ }^{2}$ School of Mechatronic Engineering and Automation, Shanghai University, Shanghai 200072, \\ China.
}

ayayang2008@live.cn, b gty@shu.edu.cn, ${ }^{\text {c }}$ zhushihaoshu@outlook.com

Keywords: RTK, SINS, Integrated Navigation, RTKLIB, AKF.

\begin{abstract}
Many situation demand a high precision, low cost and robust navigation for Land-Vehicles. Real Time Kinematic (RTK) satellite navigation is a technique that can provide up to centimeter level accuracy. But in the condition of poor visibility of satellites, the function of RTK is limited. To achieve a reliable high-precision navigation solution, this paper presents a RTK/SINS integration system based on the complementary features of RTK and SINS. The RTK is designed based on an open source program package RTKLIB. We use an innovation-based adaptive Kalman Filter with forget factor that filtering parameters can be adjusted by the change of GPS' measurement noise in our project. Finally, the reliable and high accuracy of the low cost system is verified through several experiments.
\end{abstract}

\section{Introduction}

RTK is a precise satellites navigation, which uses measurements of the phase of the signal's carrier wave, rather than the information content of the signal, can providing up to cm-level position accuracy with the help of real time corrections that provided by a single reference station or continuously operating reference stations (CORSs). It has been used in many fields such as engineering measurement and position, mobile robot, mining, agriculture, and transportation. In the open area with enough visible satellites, the performance of RTK is trustworthy. However, the accuracy of RTK may lower and even lost when in the circumstances like urban canyon, under the elevated and in the tunnel. In order to obtain a reliable result of navigation, we use IMU (inertial measurement unit) integrated with RTK/GNSS to improve the performance.

We use RTKLIB as a tool to process raw data from GPS receiver in order to acquire RTK solutions. RTKLIB is an open source program package for standard and precise positioning with GNSS developed by T. Takasu from Tokyo University of Marine Science and Technology, and distributed under the BSD 2-clause license. It is a compact and portable program library written in C to provide a standard platform for RTK-GPS applications. The library implements fundamental navigation functions and carrier-based relative positioning algorithms for RTK-GPS. It supports standard and precise positioning algorithms with GPS, GLONASS, Galileo, QZSS, BeiDou and SBAS, and also provides many library functions and APIs for GNSS data processing [1, 2].

Due to the price of Dual frequency RTK GPS receivers is too expensive, we choose the single frequency antennas and receivers in our project. While for get a high precision and robust navigation system for land-vehicles, an algorithm that integrating RTK and SINS (Strapdown Inertial Navigation System) effectively based on their respective characteristics is needed. KF (Kalman Filter) is often used to fusion the data of GPS and SINS and realize the integrated navigation system. But, the filtering accuracy will be decline even diverge if the system's measurement noise is unstable [3].

To solve the problem, we use an innovation-based adaptive Kalman Filter with forget factor that measurement noise covariance matrix and process noise covariance matrix of the system can be updated real-time in this paper. On the basis of previous studies, we designed a MEMS sensors-based RTK/SINS integrated navigation system for land-vehicles. In the second part, the model of the 
system and the method to construct it will be introduced. Then we will introduce the innovation-based adaptive Kalman Filter with forget factor proposed in this paper. At last, a series of experiment will be conducted to verify the navigation system designed in this paper.

\section{The Model of RTK/SINS Integrated Navigation System}

The signal noise rate of satellites may be changing when the vehicle moving, because the surrounding environment changing all the time, so the solution status of RTK is also changing. When the integer ambiguity is fixed and the position accuracy reaches $\mathrm{cm}$ level, we use the position and velocity which from RTK as the best solution directly and estimate the errors of other sensors. The integrated navigation mode is using RTK/SINS integrating solution as the output when the RTK solution state is single or float, while using SINS result when the satellites are invisible. Our method can select the navigation mode, estimate errors to correct the navigation parameters, and obtain the final navigation parameters effectively. The block diagram of the system is showed in figure 1.

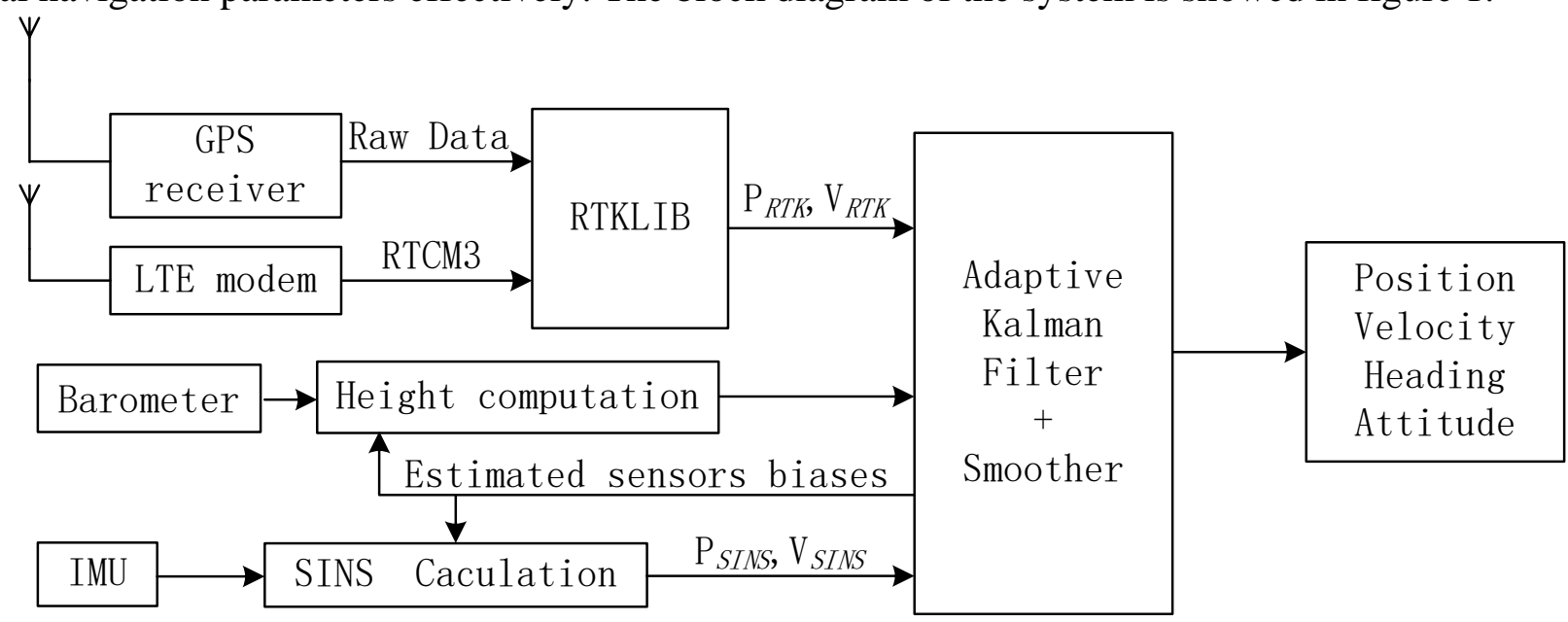

Fig. 1 The block diagram of RTK/SINS integrated navigation system

The commercial CORS (Continuous Operating Reference Stations) networks have covered various cities in china, the RTCM3 message get from CORS via 4G LTE-Modem as a data link in our project. Through an odometer can give a reliable, high precision speed information, we don't use one here because the installation is too complex. The dead reckoning method will be introduced next, which includes the selection of state vector and measurement vector, and the construction of system equation and measurement equation that describing the dynamic characteristics of the system and the relationship between the state vector and measurement vector respectively.

The State Vector and State Equation of the System. We use the E-N-U (East-North-Up) geographic coordinate system as the navigation system in this paper. The scale factor, bias errors of MEMS accelerometer and the installation angle errors are calibrated first. The speed of SINS could be calculated by mechanics equation with a variety of errors in the actual system, the real speed of SINS should be calculated by the equation below [4, 5]:

$$
\delta \dot{\mathbf{v}}_{e n}^{n}=-\boldsymbol{\varphi}^{n} \times \mathbf{f}^{n}+\delta \mathbf{v}_{e n}^{n} \times\left(2 \boldsymbol{\omega}_{i e}^{n}+\boldsymbol{\omega}_{e n}^{n}\right)+\mathbf{v}_{e n}^{n} \times\left(2 \delta \boldsymbol{\omega}_{i e}^{n}+\delta \boldsymbol{\omega}_{e n}^{n}\right)+\nabla^{n}
$$

In the equation above, superscript and subscript $n$ indicates that the parameter is represented in the navigation system. The attitude error matrix $\left(\varphi^{n} \times\right)$ is a skew-symmetric matrix with elements defined as the elements of attitude error vector $\varphi^{n}$.

After the installation angle error and the scale factor error of gyroscope is corrected, the attitude error equation of SINS is:

$$
\dot{\boldsymbol{\varphi}}^{n}=\boldsymbol{\varphi}^{n} \times\left(\boldsymbol{\omega}_{i e}^{n}+\boldsymbol{\omega}_{e n}^{n}\right)+\left(\delta \hat{\boldsymbol{\omega}}_{i e}^{n}+\delta \hat{\boldsymbol{\omega}}_{e n}^{n}\right)-\mathbf{C}_{b}^{n} \boldsymbol{\varepsilon}^{b}
$$

And the position error equation of SINS is: 


$$
\left\{\begin{array}{l}
\delta \dot{L}=\frac{\delta v_{N}}{R_{M}+h}-\delta h \frac{v_{N}}{\left(R_{M}+h\right)^{2}} \\
\delta \dot{\lambda}=\frac{\delta v_{E}}{R_{N}+h} \sec L+\delta L \frac{v_{E}}{R_{N}+h} \tan L \sec L-\delta h \frac{v_{E} \sec L}{\left(R_{N}+h\right)^{2}} \\
\delta \dot{h}=\delta v_{U}
\end{array}\right.
$$

Where, $R_{M}$ and $R_{N}$ are represent the curvature radius of the earth ellipsoid meridian and the earth prime vertical respectively. Take the latitude error $\delta L$, longitude error $\delta \lambda$, height error $\delta h$; the velocity errors $\delta v_{E}, \delta v_{N}, \delta v_{U}$ along the three directions of the navigation system; the attitude angle errors of the digital platform $\varphi_{E}, \varphi_{N}, \varphi_{U}$; the bias of gyroscope along the three directions $\varepsilon_{b x}, \varepsilon_{b y}$, $\varepsilon_{b z}$; the bias $\varepsilon_{r x}, \varepsilon_{r y}, \varepsilon_{r z}$ of gyroscope can regarded as a first-order Markov process; the bias errors of accelerometer $\nabla_{x}, \nabla_{y}, \nabla_{z}$ to compose the state vector $\mathbf{X}_{\text {SINS }}$ of SINS:

$$
\mathbf{X}_{\text {SINS }}=\left[\begin{array}{llllllllllllllllll}
\delta L & \delta \lambda & \delta h & \delta v_{E} & \delta v_{N} & \delta v_{U} & \varphi_{E} & \varphi_{N} & \varphi_{U} & \varepsilon_{b x} & \varepsilon_{b y} & \varepsilon_{b z} & \varepsilon_{r x} & \varepsilon_{r y} & \varepsilon_{r z} & \nabla_{x} & \nabla_{y} & \nabla_{z}
\end{array}\right]^{T}
$$

Then the state equation of SINS is:

$$
\dot{\mathbf{X}}_{\text {SINS }}=\mathbf{F}_{\text {SINS }} \mathbf{X}_{\text {SINS }}+\mathbf{G}_{\text {SINS }} \mathbf{W}_{\text {SINS }}
$$

Take the rest term of SINS' error equations to compose matrix $\mathbf{F}_{N}$, then the state matrix is:

$$
\mathbf{F}_{\text {SINS }}=\left[\begin{array}{cc}
\mathbf{F}_{N} & \mathbf{F}_{S} \\
\mathbf{0} & \mathbf{F}_{M}
\end{array}\right]_{18 \times 18}
$$

Where,

$$
\mathbf{F}_{S}=\left[\begin{array}{ccc}
\mathbf{C}_{b}^{n} & \mathbf{C}_{b}^{n} & \mathbf{0}_{3 \times 3} \\
\mathbf{0}_{3 \times 3} & \mathbf{0}_{3 \times 3} & \mathbf{C}_{b}^{n} \\
\mathbf{0}_{3 \times 3} & \mathbf{0}_{3 \times 3} & \mathbf{0}_{3 \times 3}
\end{array}\right]_{9 \times 9}
$$

The Measurement Vector and Measurement Equation of the System. Take the position and velocity differences between SINS and RTK as the measurement vector, then can obtain the measurement equation:

$$
\mathbf{Z}(t)=\left[\begin{array}{l}
\mathbf{P}_{\text {SINS }}-\mathbf{P}_{R T K} \\
\mathbf{v}_{\text {SINS }}-\mathbf{v}_{R T K}
\end{array}\right]=\mathbf{H}(t) \mathbf{X}(t)+\mathbf{V}(t)
$$

Where $\mathbf{P}_{\text {SINS }}$ and $\mathbf{v}_{\text {SINS }}$ represent the position and velocity measurement value of SINS respectively, which including the error massages along the three directions of the navigation system; $\mathbf{P}_{R T K}$ and $\mathbf{v}_{\text {RTK }}$ represent the position and velocity measurement value of RTK respectively. The measurement output matrix $\mathbf{H}(t)$ is:

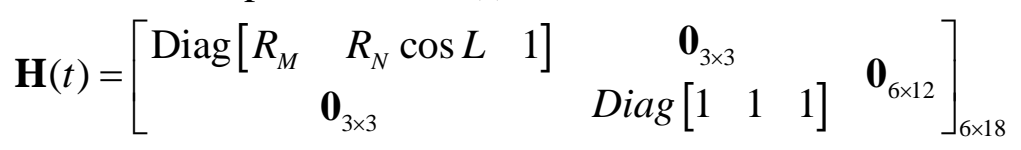

The Adaptive Kalman Filter. The filter model of the RTK/SINS integrated navigation system is introduced above, and the specific steps of this algorithm is [6]:

(1) Estimation of the system's state vector:

$\delta \hat{\mathbf{X}}_{k / k-1}=\boldsymbol{\Phi}_{k, k-1} \delta \hat{\mathbf{X}}_{k-1}$

(2) The calculation of mean square error of the prediction:

$\mathbf{P}_{k / k-1}=\boldsymbol{\Phi}_{k, k-1} \mathbf{P}_{k-1} \boldsymbol{\Phi}_{k, k-1}^{T}+\mathbf{Q}_{k-1}$ 
(3) When the solution status of RTK is fixed or the measurement noise of GPS is small, The calculation of adaptive matrix $\mathbf{R}_{k}$ based on the innovation sequence:

$$
\mathbf{R}_{k}=\frac{1}{n} \sum_{i=0}^{n-1} \delta \mathbf{Z}_{k-i} \delta \mathbf{Z}_{k-i}^{T}-\mathbf{H}_{k} \mathbf{P}_{k / k-1} \mathbf{H}_{k}^{T}
$$

Where, $\delta \mathbf{Z}_{k-i}$ is the difference between the estimation value and the measurement value at each time and is defined as the innovation sequence; $\sum_{i=0}^{n-1} \delta \mathbf{Z}_{k-i} \delta \mathbf{Z}_{k-i}^{T}$ is the sum of autocorrelation matrix of the innovation sequence and $n$ is the number of samples.

Meanwhile, back up the matrix sequence $\mathbf{R}_{k}$ as:

$$
\mathbf{R}_{b a k}=\mathbf{R}_{k}
$$

When the signal of satellites is poor or missing, the conventional EKF is used with the system saved and fixed measurement noise covariance matrix.

(4) Filtering gain updates:

$\mathbf{K}_{k}=\mathbf{P}_{k / k-1} \mathbf{H}_{k}^{T}\left[\mathbf{H}_{k} \mathbf{P}_{k / k-1} \mathbf{H}_{k}^{T}+\mathbf{R}_{k}\right]^{-1}$

(5) The estimation of state vector:

$$
\delta \hat{\mathbf{X}}_{k}=\delta \hat{\mathbf{X}}_{k / k-1}+\mathbf{K}_{k}\left[\delta \mathbf{Z}_{k}-\mathbf{H}_{k} \delta \hat{\mathbf{X}}_{k / k-1}\right]
$$

(6) The mean square error of the estimation:

$$
\mathbf{P}_{k}=\left[\mathbf{I}-\mathbf{K}_{k} \mathbf{H}_{k}\right] \mathbf{P}_{k / k-1}\left[\mathbf{I}-\mathbf{K}_{k} \mathbf{H}_{k}\right]^{T}+\mathbf{K}_{k} \mathbf{R}_{k} \mathbf{K}_{k}^{T}
$$

(7) The estimation of adaptive matrix $\mathbf{Q}_{k}$ online with forgetting factor:

$$
\widehat{\mathbf{Q}}_{k}=\left(1-d_{k}\right) \widehat{\mathbf{Q}}_{k-1}+d_{k} \frac{1}{L-1} \sum_{k=1}^{L}\left[\left(\delta \mathbf{Z}_{k}-\overline{\delta \mathbf{Z}}\right)\left(\delta \mathbf{Z}_{k}-\overline{\delta \mathbf{Z}}\right)^{T}-\frac{L-1}{L}\left(\Phi_{k, k-1} \mathbf{P}_{k-1} \boldsymbol{\Phi}_{k, k-1}^{T}-\mathbf{P}_{k}\right)\right]
$$

Where, $d_{k}=(1-b) /\left(1-b^{k}\right) b$ is called the forgetting factor and typically has a positive value between 0.98 and 0.995. $L$ is the number of measurement noise samples, $\overline{\delta \mathbf{Z}}$ is the unbiased estimation of $\delta \mathbf{Z}_{k}$. In our method, we tread $\mathbf{Q}_{k}$ as a constant vector at first. When the innovation sequences is enough, the $\mathbf{Q}_{k}$ will be updated every step. The limited length of forgetting factor can improve the weight of the recent samples so that the navigation parameters can be updated in time.

The algorithm above can use the innovation sequence to estimate the process noise covariance matrix $\mathbf{Q}_{k}$ and measurement noise covariance matrix $\mathbf{R}_{k}$ effectively with the help with the result of RTK when the solution status of RTK is fixed or the measurement noise of GPS is small. Meanwhile, the filtering parameters is adjusted with the change of GPS' measurement noise that can be acquired a high precision and robust navigation solution.

\section{Land-Vehicle Tests}

\section{The Test Equipment and Conditions.}

According to the method described above, we conducted an experiment to verify and evaluate the performance of the proposed INS/RTK-GPS integration scheme. The hardware design that we have used in the project are including a commercial single signal frequency GNSS receiver U-Blox Neo-M8T, a tri-axial accelerometer SCC1300-D04, a tri-axial gyroscope STIM202. The process of RTK and integrated navigation algorithm is loaded in our development board. The development board has a 1000MHz ARM Cortex-A8 core CPU (Samsung S5PV210), 512MB DDR2 RAM, 1GB flash and supports embedded Linux. We connect some peripherals like WIFI model, Bluetooth, USB ports and serial interfaces on the board to communicate with other PCB units and CORS. In order to facilitate the experiment, we add an ARM Cortex-M4 core MCU STM32F407 and SD card to save operational log and raw data for the post processing. 
The test starts in the environment without shelter. Then we drive in many complex environment like urban canyon, under the elevated and though the tunnel. The signal of satellites can be affected by the multipath effect and shelter, so the solution status of RTK is changing depend on the surrounding environment. The average speed of the vehicle is about $55 \mathrm{~km} / \mathrm{h}$.

The Test Result and Analysis. The track in picture 2 is the trajectory when driving two circles around one high building that located in the business district. There are many hundreds meters high buildings that the surface is covered with glass. In the figure 2 the blue locus is generated by the RTK-GPS' data, and the red one is the RTK/SINS integrated navigation system's data. The result shows that the RTK has been affected by the multipath effect and the signal of satellites, but the integrated navigation system designed in this paper can calculate the position correctly.

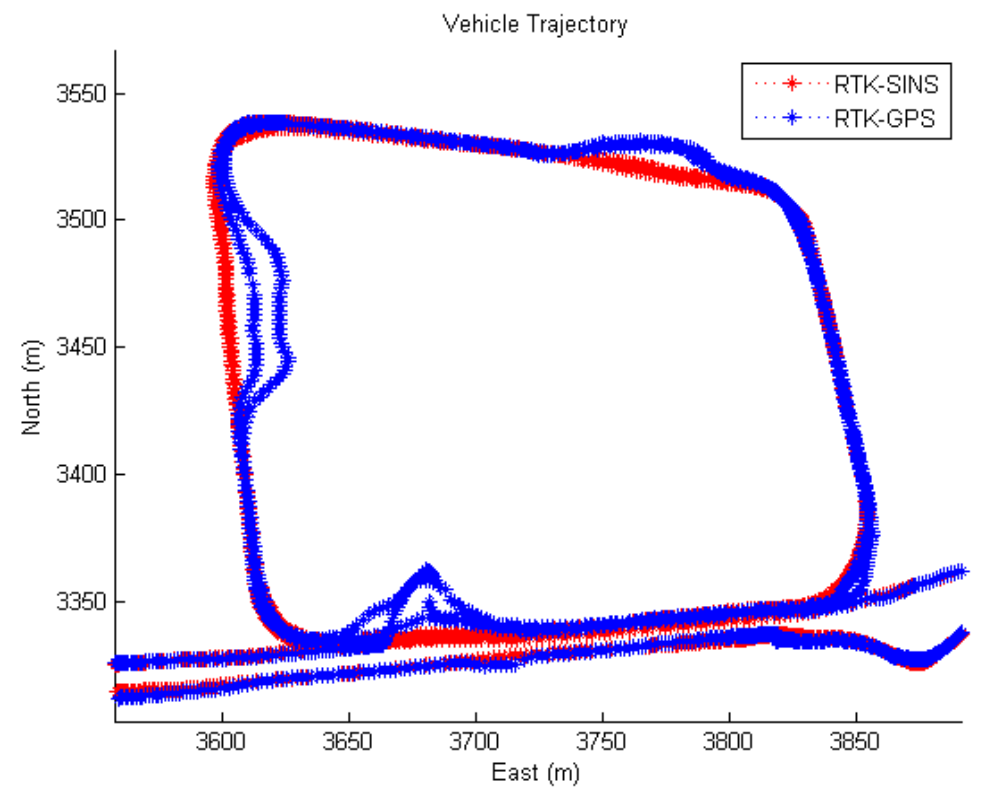

Fig. 2 The traveling trajectory of vehicle in urban canyon

In order to make the results more obvious, we convert the result data (files) above to KML for Google Earth to display the result on a real map. We can see the trajectories showed in figure 3 on 3D maps by Google Earth. The blue locus is RTK-GPS' data and the green one is RTK. The trajectories of RTK have deviated from the real road on map, the solution of integrated navigation is accuracy so that can be matched the real roads much better.

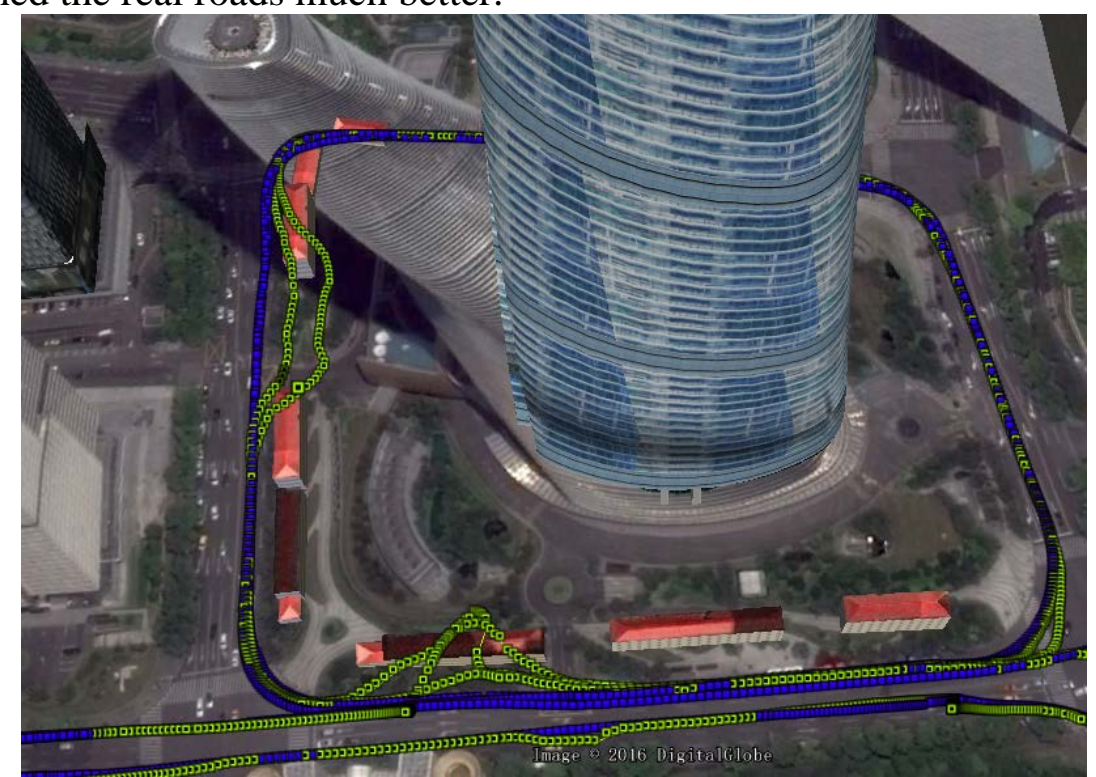

Fig. 3 The traveling trajectory on the 3D Map of Google Earth

When came back, the vehicle had gone through a tunnel underwater. Just enter the tunnel, the signal of satellites was missing. The length of the tunnel is about $1500 \mathrm{~m}$ and the average speed of the 
vehicle is about $30 \mathrm{~km} / \mathrm{h}$. The trajectory can be seen in the figure 4 (from right to left). When re-acquisition of the satellite signals, the solution error between RTK and SINS is very small, which means the result of SINS is reliable.

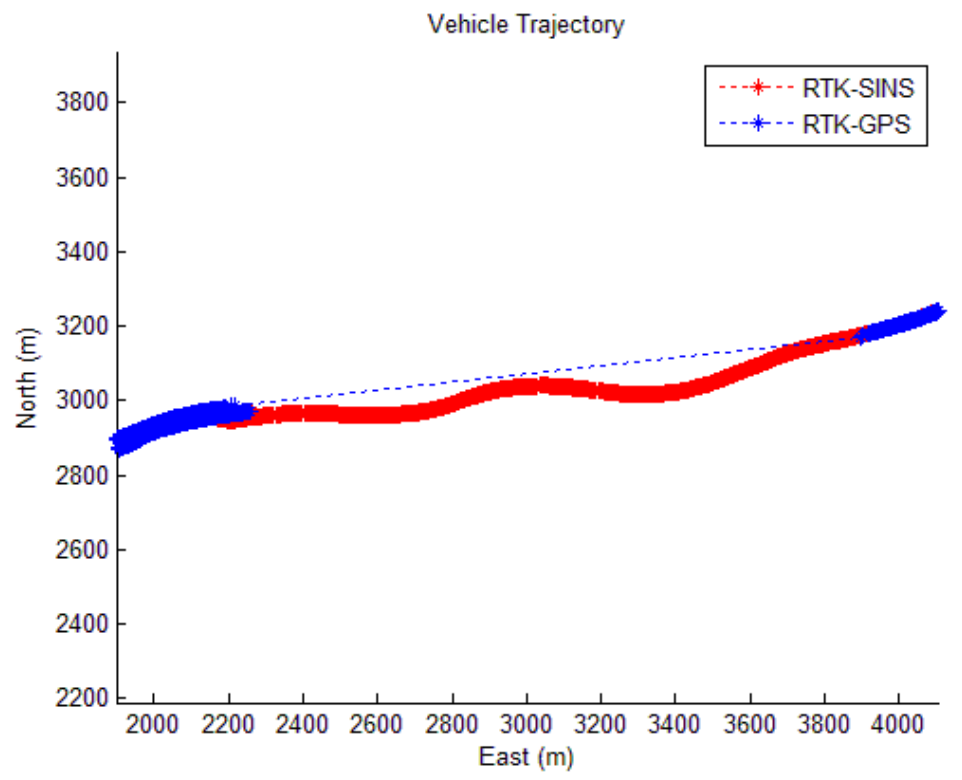

Fig. 4 The trajectory of go through tunnel underwater

\section{Summary}

In this paper, we designed a high precision, low cost and robust navigation system for land-vehicles including the hardware and software. The RTK is based on the open source program package RTKLIB. Based on the complementary features of RTK-GPS and SINS, we designed an innovation-based adaptive Kalman Filter with forget factor as the RTK/SINS integrated navigation algorithm, which uses the innovation sequence to estimate the measurement noise covariance matrix and process noise covariance of the system in real-time. The limited length of forgetting factor can improve the weight of the recent samples so that the navigation parameters can be updated in time. The test result shows that our project and method can give a high precision, reliable navigation solution.

\section{References}

[1] T.Takasu, A.Yasuda, Development of the low-cost RTK-GPS receiver with an open source program package RTKLIB, 2009 international symposium on GPS/GNSS, International Convention Centre Jeju, Korea, 2009

[2] T. Takasu, RTKLIB ver. 2.4.2 Manual, 2013. http://www.rtklib.com/prog/manual_2.4.2.pdf

[3] P. Davidson, J. Hautamäki, J. Collin and J. Takala, Improved vehicle positioning in urban environment through integration of GPS and low-cost inertial sensors, Proceedings of the European Navigation Conference, 2009.

[4] Q. Y. Yuan, Inertial Navigation, second edition, Science Press, BeiJing, 2014.

[5] D. H. Titterton and J. L. Weston. Strapdown inertial navigation technology, volume 17. Institution of Engineering and Technology, 2004.

[6] Z. S. Hao, G .T. Yue, C.Y. Yang, GPS/SINS integrated navigation system using an innovation based adaptive Kalman Filter for land-vehicles. Energy Science and Applied Technology ESAT 2016, pp.507-510. 